Anna Kapuścińska

Uniwersytet Kazimierza Wielkiego w Bydgoszczy

(iD https://orcid.org/0000-0001-6316-1838

\title{
Mensch oder Computer? Zur Frage des Produzenten in den modernen visuellen Medien
}

\author{
The Human or the Computer? \\ On the Question of the Producer in Modern Visual Media \\ Człowiek czy komputer? \\ Pytanie o producenta we współczesnych mediach wizualnych
}

\begin{abstract}
The aim of the article is to reflect on the role of humans as producers of information in the mass media. Even if they are still present in the process of information production, they cannot always be considered as producers in the communication process. Their role is often limited to three activities: manufacturing the computer, pre-programming it and typing the new content into a pre-programmed design scheme. However, it can be argued that none of these activities is directly connected with producing the information. This allows an assumption that the actual information production beyond the activity of a human producer and may rather be seen as the machine reconstruction of a pre-defined scheme.
\end{abstract}

Key words: mass media, communication, text design, information

\begin{abstract}
Abstrakt: Ziel des Artikels ist es, über die Rolle des Menschen als Produzenten von Informationen in den Massenmedien zu reflektieren. Selbst wenn er im Prozess der Informationsproduktion nach wie vor vorhanden ist, kann er nicht immer als Produzent im Kommunikationsprozess betrachtet werden. Seine Rolle beschränkt sich häufig auf drei Aktivitäten: Herstellung des Computers, seine Vorprogrammierung und Eingabe des neuen Inhalts in ein vorprogrammiertes Gestaltungsschema. Es kann jedoch argumentiert werden, dass keine dieser Aktivitäten direkt mit der Erzeugung der Informationen verbunden ist. Dies erlaubt die Annahme, dass die tatsächliche Informationsproduktion über die Tätigkeit eines menschlichen Produzenten hinausgeht und eher als maschinelle Rekonstruktion eines definierten Schemas angesehen werden sollte.
\end{abstract}

Schlüsselwörter: Massenmedien, Kommunikation, Teytdesign, Information

\begin{abstract}
Abstrakt: Celem artykułu jest refleksja nad rolą czynnika ludzkiego przy produkcji informacji $w$ środkach masowego przekazu. Nawet jeśli człowiek nadal uczestniczy w procesie tworzenia informacji, nie zawsze można uznać go za producenta $w$ procesie komunikacji. Jego rola jest często ograniczona do trzech czynności: wyprodukowania komputera, jego wstępnego zaprogramowania i wpisania nowej treści do wcześniej zaprogramowanego schematu graficznego. Można jednak stwierdzić, że żadna z tych czynności nie jest bezpośrednio związana z wytwarzaniem informacji, co oznacza, że faktyczna produkcja informacji wykracza poza aktywność człowieka jako producenta i może być raczej postrzegana jako maszynowa rekonstrukcja określonego uprzednio schematu.
\end{abstract}

Słowa kluczowe: media masowe, komunikacja, design tekstu, informacja 


\section{Problemgegenstand und Zielsetzung}

Der Grundgedanke des vorliegenden Beitrags ist die übliche Annahme, dass die primäre Funktion der modernen visuellen Medien mit Kommunikation verbunden ist. Dabei wird von dem Konzept des Kommunikationsprozesses nach Umberto Eco ausgegangen, wonach der Kommunikationsprozess dann vorliegt, „wenn ein Sender bewußt kodierte Signale mittels eines Sendegerätes überträgt, das sie über einen Kanal schickt" (Eco, 1977: 167).

Es wird hierbei der Frage nachgegangen, ob man es in den modernen visuellen Medien tatsächlich mit der Kommunikation zu tun hat, und zwar, ob die beiden Bedingungen: des menschlichen und bewussten Senders erfüllt sind. Anders formuliert, konzentrieren sich die vorliegenden Überlegungen auf die Identität des Produzenten der Kommunikate, die in den modernen visuellen Medien unterbreitet werden. Eine ähnliche Fragestellung ist bereits in Bezug auf die Nachrichtenticker diskutiert worden (ugl. KAPuścıŃSKA, 2017). Die in diesem Beitrag präsentierten Thesen beschränken sich jedoch nicht nur auf dieses einzelne Element und auch nicht nur auf das Medium Fernsehen. Dieser Text hat vielmehr einen allgemein theoretischen Charakter und die darin angeführten Beispiele entstammen keiner korpusbasierten Analyse, sondern dienen lediglich zur Illustrierung der theoretischen Thesen.

Die Frage nach einem menschlichen (und dabei auch bewussten) Sender lässt über die Rolle des Menschen bei der Produktion der medialen Kommunikate reflektieren. Jedenfalls kann die Kommunikation in den modernen Medien ohne den Menschen nicht zustande kommen. Er ist derjenige, der zumindest drei Tätigkeiten ausführt, die für die Kommunikation relevant sind: Er stellt die elektronischen Geräte her, programmiert sie (entsprechend einem gegebenen Gestaltungsschema der Kommunikation) und führt einzelne aktuelle Mitteilungen ein. Nun ist jedoch zu diskutieren, welche (wenn überhaupt) von diesen Tätigkeiten tatsächlich zum Kommunikationsprozess (in der hier postulierten Bedeutung) gehören.

\section{Kommunikation und Erzeugung elektronischer Geräte}

Die Erzeugung der technischen Geräte wie Computer, sowie auch aller anderen, weniger technisch fortgeschrittenen Gegenstände, die im Nachhinein zur Kommunikation gebraucht werden können, kann nur schwer als ein Teil des Kommunikationsprozesses aufgefasst werden. Dieses Verhältnis lässt sich anhand der von Roland Posner geführten Diskussion erklären. Somit sind solche Gegenstände als Artefakte zu betrachten, weil sie - ausgenommen z. B. natürliche Federn, die in der Geschichte zum Schreiben dienten - von Menschen erzeugt sind (ugl. Posner, 1994: 18). Erst wenn man das Artefakt zu einem bestimmten Zweck verwendet, macht man es zum Instrument. Folgerichtig, wenn man mit dem Computer ein Kommunikat erzeugt, macht man ihn zum Instrument der Kommunikation, dessen Erzeugung jedoch weiterhin nicht als ein Bestandteil des Kommunikationsprozesses zu betrachten ist. 
Die zweite Fragestellung betrifft den Prozess des Programmierens, indem in das Bedienungssystem des Computers die Vorgaben über die visuelle Gestaltung der emittierten Kommunikate eingeführt werden. Die auf diese Weise erzeugten Schemata von Internetseiten, Fernsehsendungen usw. entsprechen sowohl den allgemein etablierten Mustern für die jeweiligen Kommunikationsarten als auch den Mustern, die einer bestimmten "Marke“ wie ein bestimmtes Internetportal oder eine bestimmte Sendung eigen sind (ugl. KAPUŚCıŃSKA, 2020: 99).

Die ersteren knüpfen im Grunde genommen auf das Muster der „Mosaikartigkeit“ an, das bereits von Marshall MCLuHAN (1995: 312; ugl. dazu auch KAPuśCıŃSKA, 2017: 173) beschrieben wird. McLuhan zufolge ist der Ursprung solcher Konstruktion in der Gewohnheit der Empfänger zu einer spezifischen Art der Wahrnehmung zu sehen, die durch die Popularisierung des Fernsehens bedingt sein soll. Auch die mosaikartig gestaltete Presse wie „Time“ oder „Newsweek“ soll durch den Einfluss des Fernsehens ihre Auflagen verdoppelt haben. Es wird von McLuhan mit der fernsehbedingten Verschiebung der Funktion von der Presse begründet. Während die „älteren Illustrierten“ ein „Fenster zur Welt“ öffnen, geben die neueren nach McLuhan stattdessen „Gruppenleitbilder der lebendigen Gesellschaft“ (McLuhan, 1995: 312). McLuhan macht den Zusammenhang deutlich, der zwischen der Ausbreitung dieser Tendenz und dem technischen Fortschritt bereits lange vor Erfindung der Elektrizität besteht. Die Ursprünge davon sieht er bereits in der Einführung der mit Dampf betriebenen Rotationspresse (ugl. McLuhan, 1995: 313).

Die dadurch entfachte technische Beschleunigung hat auch die Anforderungen des Publikums geprägt, die schnellstmöglich über das Aktuelle informiert werden wollte. Die „Automation“ hat nach McLuhan bloßgestellt, dass die Medien wie Radio, Presse oder Fernsehen nicht - wie manche glaubten - rein Informationsformen sind, "die von den Herstellern und Benutzern von »Artikeln« wie Autos, Seife und Benzin bezahlt würden“ (McLuhAN, 1995: 313). Die Information an sich musste seither vielmehr als "die entscheidende Handelsware“ betrachtet werden, während die materiellen Waren nur als „Begleiterscheinungen der Informationsbewegung galten“ (MCLUHAN, 1995: 315-316).

Aus der Perspektive des behandelten Themas lässt sich davon ausgehen, dass die fortschreitende Automatisierung die Rolle des menschlichen Senders zunehmend übernommen hat. Während die ganze Nachricht früher manuell von einem Menschen gesetzt werden musste, konnte dies infolge des technischen Fortschritts durch eine Maschine übernommen werden. Zwar handelte es sich bis zur Erfindung des Computers noch nicht um die Programmierung im engen Sinne, dafür aber um eine entsprechende Einstellung der Maschine. Dies ähnelt dem Beispiel der „Kommunikation“ zwischen Maschinen, das von Umberto Eco angeführt wird. Wenn ein Sendegerät ein Signal z. B. über das Erreichen des Wasserspiegels an das Empfangsgerät sendet, ist das Empfangsgerät „entsprechend instruiert, dass [es] beim Empfang der Botschaft eine Korrektur der Ausgangssituation auslöst (z. B. einen feedback-Mechanismus, der die Wasserentleerung im Becken sorgen“ (Eco, 2002: 48). Dieses „Instruieren“ des Gerätes kann man mit dem Programmieren des Computers vergleichen. Bemerkenswert ist jedoch, dass Eco nicht den „Instruierenden“, sondern das Gerät als den Sender bei dieser „Kommunikations“-Situation betrachtet. Auch wenn es umstritten ist, ob 
man bei der „Kommunikation“ zwischen Maschinen tatsächlich von der Kommunikation sprechen soll, unterliegt keinem Zweifel, dass das Instruieren bzw. Programmieren der Maschine, die dadurch „lernt“, wie sie auf eine gegebene Situation reagieren soll, nach Eco nicht zum Kommunikationsprozess gehört. Die Sendefunktion wird damit nur dem maschinellen Element und nicht dem agierenden Menschen zugeschrieben.

\section{Kommunikation und Eingabe von Informationen}

Der dritte Aspekt, der über die Rolle des Menschen bei der Kommunikation in den „neuen Medien“ reflektieren lässt, ist der Prozess der Einführung von jeweils neuen Informationen in die vordefinierten Schemata. Auf die Bedeutung des Zusammenspiels zwischen dem Neuen und dem Vertrauten in den Fernsehnachrichten wird von Ulrich Schmitz hingewiesen. Demnach nähren sie sich „aus einem ewig neuen Widerspiel von erwarteter Überraschung und irritierter Gewohnheit“ (Sснмıтz, 2004: 50). Die Tendenz, die am Beispiel der Fernsehsendungen sichtbar ist, ist jedoch zugleich ein Merkmal der gesamten visuellen Kommunikation in den Massenmedien. Obwohl sich diese Medien nicht nur voneinander deutlich unterscheiden, sondern auch in sich nicht homogen sind, ist ihnen die Anwesenheit vorprogrammierter Schemata gemeinsam. Dieser Schematismus habe zum Ziel, die Sendung zu einem „Ritual“ umzuwandeln, „das Umweltkomplexität reduziert und Kommunikation synchronisiert" (Schmiтz, 2004: 50). Nach Schmitz zieht

[d]er in kurzen Rhythmen dauernd erneuerte Aktualitätszwang [...] routinierte Verfahren (sowohl in der Realität als auch in der Berichterstattung) nach sich, mit denen Geschehen in Ereignisse verwandelt wird. Sieht man von inderikalischen Variablen (als Eigennamen, Zahlen, Orts- und Zeitangaben) ab, so hätte ein großer Teil der Nachrichtentente etwas dieses heutigen Tages auch vor fünf oder vielleicht fünfundzwanzig Jahren veröffentlicht werden können.

SCHMITZ, 2004: 50-51

Diese Behauptung lässt sich anhand von einer völlig zufällig ausgewählten Nachrichtensendung im deutschen Fernsehen - ZDF heute am 13. November 2020 um 19.00 Uhr - bestätigen. Obwohl die Sendung aus der Zeit der Coronavirus-Pandemie stammt, die an sich eine beispiellose Situation ist, sieht man überraschenderweise auch darin Spuren der von Schmitz antizipierten Routiniertheit.

Sie lässt sich bereits in einem von drei Themen des Nachrichtenüberblicks beobachten. Es betrifft die Abweisung der Vorwürfe auf einen Wahlbetrug bei den Präsidentenwahlen in den Vereinigten Staaten. Die Nachricht folgt tatsächlich einem fest etablierten und zeitlosen Schema. Die Vorwürfe gegen Wahlbetrüge werden oft in verschiedenen demokratischen Ländern erhoben und in der Regel abgelehnt.

Darauf folgt als eine Aufmachermeldung die Information über die schwierige Situation im Pflegesektor. Wie auch die Moderatorin (Barbara Hahlweg) selbst betont: „Schon lange vor Corona war klar, was jetzt erst recht ans Licht kommt“. Von dem Problem des Mangels an Pflegekräften wurde also zweifelsohne schon mehrmals berichtet, u. a. vor zwei Jahren 
als sich - der Moderatorin zufolge - „die beteiligten Ministerien, Verbünde und Krankenkassen zu konzertierten Aktion Pflege zusammen[schlossen]“. Die aktuelle Nachricht beziehe sich auf die erste Bilanz dieser Aktion, von in einer Bundespressekonferenz berichtet wird. Daran lässt sich die von Schmitz signalisierte Tendenz erkennen, Geschehen in Ereignisse umzuwandeln (ugl. Scнmitz, 2004: 51), denn der Moderatorin zufolge sei sie „noch nicht am Ziel, aber auf dem Weg dahin, zu mehr Stellen, einer besseren Bezahlung und einer guten Ausbildung".

Auch die sechste Nachricht, die von dem Gedenken an die Terroropfer fünf Jahre nach dem Attentat in Paris handelt, zeigt die Tendenz zur Zeitlosigkeit. Sicherlich wird jedes Jahr der Opfer gedacht und daher könnte eine solche Mitteilung am 13. November eines beliebigen Jahres ausgestrahlt werden, und zwar umso mehr, weil darin zum großen Teil Archivuideos angezeigt werden.

Noch ein anderes Beispiel ist die achte (und zugleich vorletzte) Nachricht zum Thema der illegalen Grenzüberschreitungen von Flüchtlingen. Zwar handelt es sich in diesem konkreten Fall um die Situation an der Grenze zwischen Kroatien und Bosnien-Herzegowina, aber das Problem kommt bereits seit fünf Jahren mehrmals an verschiedenen EU-Grenzen vor.

Abgesehen von dem inhaltsbezogenen Schematismus wird in den Nachrichtensendungen auch ein grafisch-akustisches Schema realisiert. Seine Komponenten schaffen einen Rahmen, der für den „Komfort“ des Empfängers sorgt. Nach Neil Postman: „Würde die Musik fehlen [...], so würden die Zuschauer denken, es sei etwas wirklich Beunruhigendes, womöglich ihr Leben Veränderliches geschehen. Aber solange die Musik der Sendung einen Rahmen gibt, darf sich der Zuschauer in dem Glauben wiegen, daß nichts geschehen ist, worüber er sich ernstlich aufregen müsste“ (Postman, 2008: 127).

Auch diese Ansicht lässt sich mit dem Beispiel von ZDF heute untermauern. Die Musik ist darin bei dem Vorspann, dem Nachrichtenüberblick, der Eröffnung (mit der Stimme im Off: „Die Nachrichten des Tages, heute mit Barbara Hahlweg“) sowie dem Abschluss. Neben der Hintergrundmusik, die bei allen diesen Strukturelementen zu hören ist, lassen sich zwei Jingle-Signale unterscheiden. Der erste wird drei Mal: beim Vorspann, bei der Eröffnung und beim Abschluss abgespielt. Der zweite, leisere ertönt während des Nachrichtenüberblicks, am Anfang jeder einzelnen Meldung.

Da es sonst keine Musik während der Sendung gibt, passt dies besonders gut zu der von Postman angeführten Metapher des Theaters, indem dieser Rahmen (vergleichbar einem Vorhang) die News wie „die Szenen eines Theaterstücks“ (Postman, 2008: 128) betrachten lässt.

Zu der Metapher des Theaterstücks passt ebenso die grafische Gestaltung. In der Regel folgt aber nicht nur die Nachrichtensendung, sondern das ganze Fernsehprogramm einem vordefinierten Schema, das zum großen Teil die identifizierende Funktion hat und dadurch zu einem „Markenzeichen“ des Programms wird. Bei dem Fernsehprogramm ZDF gibt es zumindest zwei explizite Bindeelemente. Das eine ist die tradierte Markenfarbe - Orange - nicht nur in der Senderlogotype, sondern auch in den Gestaltungselementen von den einzelnen Sendungen sowie dem Internetauftritt des Programms. Das andere sind die Gestalten der Mainzelmännchen, die als Sender-Maskottchen gelten. Diese erscheinen nicht nur in kurzen Zeichentrickfilmen in der Zwischenzeit zwischen den Sendungen als eine Einleitung für die Werbung, sondern auch als unbewegte Bilder im Internet (darunter als Illustration 
für die Wettervorhersage auf der Internetseite der ZDF-Nachrichtensendung Heute). Sie sind inzwischen zu einem Kulturphänomen geworden. In Verkauf sind unterschiedliche Gadgets mit dem Motiv vom den Mainzelmännchen (wie Becher, Schlüsselanhänger oder T-Shirts) - jeweils in Verbindung mit der ZDF-Logotype und auf einigen Verkehrsampeln in Mainz sind die klassischen Ampelmänner mit Mainzelmännchen ersetzt.

Derartiger Schematismus beschränkt sich jedoch nicht nur auf das Fernsehen. Auch z. B. in Zeitungen werden in der Regel die jeweils neuen Inhalte in das vorgegebene Schema inskribiert. Als ein Beispiel dafür kann „Frankfurter Allgemeine Zeitung“ angeführt werden. Die Betrachtung einiger zufälliger Ausgaben lässt feststellen, dass sich der Schematismus nicht nur auf die konsequente Verwendung der modularen statt einer buchartigen Struktur beschränkt. Vor allem auf den ersten Seiten der Zeitungen fällt es auf, dass sie nahezu identisch strukturiert sind. Die Tente sind nicht nur gleich verteilt, sondern nehmen auch jeweils genauso viel Platz auf, wodurch auch die Abgrenzungslinien zwischen ihnen genau gleich positioniert sind. Diese Beobachtung macht deutlich, dass selbst der Umfang der einzelnen Texte dem festen Schema unterliegt. Die einzige sichtbare Variabilität in der Struktur der Seite betrifft die Abgrenzungslinie zwischen dem Aufmacherbild und dem tertuellen Aufmacher. In manchen Ausgaben kommt es vor, dass die beiden Elemente zu einer inhaltlichen Einheit verbunden sind, und in diesem Fall wird auf die horizontale Linie zwischen ihnen verzichtet.

Nur die Terte, die in dieses Schema jeweils eingegeben werden, sind eindeutig als ein Erzeugnis des Menschen zu betrachten. Im Zusammenhang mit der Frage nach dem menschlichen Hinzutun bei einem solchen Kommunikationsprozess ist daher ausschlaggebend, was man als das eigentliche Kommunikat betrachtet: die gewohnte grafische Gestaltung oder die jeweils neu eingegebenen einmaligen News.

Selbst durch die Erscheinungsform von dem Schema wird eine Reihe von Informationen vermittelt (ugl. dazu KAPuścıŃSKA, 2020: 99). Neben der Örtlichkeit der einzelnen Elemente auf dem Papierblatt bzw. der Bildschirmfläche trägt dazu auch die spezifische Gestaltungsform bei, die aufgrund von dem Vorwissen des Rezipienten erkennen lässt, dass es sich um eine bestimmte Art der Fernsehsendung (wie z. B. Nachrichtensendung), um eine Zeitung, Zeitschrift oder Internetseite handelt. Alle diese Kommunikationsmedien haben eine bestimmte grafische Struktur, die über ein bestimmtes Fernsehprogramm, eine bestimmte Presseagentur oder einen Inhaber der Internetseite hinausreicht. Ferner lässt die Gestaltung eine Untergruppe identifizieren. Es gibt nämlich auch solche Elemente des Musters, die z. B. nicht alle Nachrichtensendungen kennzeichnen. Das sieht man z. B. in den Unterschieden zwischen einer Nachrichtensendung bei einem staatlichen Programm wie ZDF oder ARD und bei einem Nachrichtenprogramm, z. B. n-tu. In der zweiten kommen zusätzliche Gestaltungselemente vor, wodurch die Sendungen in einem noch höheren Grad den Charakter einer Multiübertragung erlangen (ugl. CieszKowsKı, 2014).

Dasselbe gilt auch für andere Medien wie die Presse. Obwohl die Binnendifferenzierung zwischen der Boulevardpresse und der sog. „Qualitätspresse“ heutzutage zum großen Teil verschwommen ist, gibt es allerdings immer noch Merkmale, die beide Arten voneinander unterscheiden. Trotz der fortschreitenden Boulevardisierung der Qualitätspresse wird die Mosaikartigkeit mit dem Vorrang bildlicher Elemente nach wie vor meistens als ein Kennzeichen der Boulevardpresse betrachtet. 
Andererseits ist die Frage zu stellen, was die vom Menschen eingeführten aktuellen Inhalte kommunizieren (können). Selbstuerständlich ist in den sprachlich kodierten Kommunikaten jeweils ein Inhalt enthalten, der unter der Annahme, dass der Empfänger der gegebenen Sprache mächtig ist, dekodiert werden kann. Mit anderen Worten sind sie kommunikative Zeichen (ugl. Posner, 1994: 16). In der Pranis ist ausgerechnet die Anwesenheit der oben diskutierten Gestaltungsschemata ein wichtiger Faktor, der diese Rezeption, und folgerichtig auch das Zustandekommen der Kommunikation, verhindert, weil sie nur dann möglich ist, wenn „es dem Adressaten gelingt, aufgrund des Zeichens die Botschaft zu rekonstruieren“ (ugl. Posner, 1994: 16). Wie zahlreiche Studien ergeben, bewirkt die dadurch bedingte Gleichzeitigkeit und Geschwindigkeit ihrer Darstellung die Überforderung des menschlichen Perzeptionsuermögens und die Wiederholbarkeit - Langeweile (ugl. KapuśCińSKA, 2016: 27, CieszKowski, 2014, Trojanowska \& Francuz, 2007: 68). Ebenso wie für die sprachliche Komponente der Nachrichtenticker, gilt auch für andere sprachliche Komponenten der massenmedialen Kommunikation, dass sie zum großen Teil austauschbare Komponenten der Schemata sind. Ihre kommunikative Funktion kann als erfüllt gelten, wenn sie beim ersten Blick, d. h. bei einer bildspezifischen Betrachtung der gesamten "Sehfläche“ (ugl. Schmitz, 2011) zu dem Schema passen.

Dafür müssen zwei Bedingungen erfüllt werden. Die eine betrifft die typografischen Eigenschaften wie die Art der Schrift, ihre Größe und Farbe, die ebenso ein Element des „Marken“-Schemas sein kann. Als ein Beispiel dafür lässt sich die Zeitung „Bild“ anführen. Die darin gebrauchte Schrift der Schlagzeilen ist eine serifenlose Schrift mit fetten Buchstaben (ugl. KAPuŚCıŃSKA, 2012: 28). Dadurch sind die Schlagzeilen nicht nur selbst bei einer Entfernung auffallend, sondern auch korrespondieren sie mit der Schriftart, die in der Logotype der Zeitung gebraucht wird. Auch die Farben der eingesetzten Schrift sind nicht beliebig. Dies ist in der Regel eine Kombination von zwei Farben: schwarz und weiß oder von drei Farben: schwarz, weiß und rot, wobei die rote Farbe oft auf eine sonst ungewöhnliche Weise in der Unterstreichung andersfarbiger Buchstaben vorkommt. Unbestreitbar gehört die Typografie bei stark schematisierten Darstellungen zu wichtigen Gestaltungselementen. Damit wird die "Marke“ kommuniziert, die durch eine kommunikative Einheit vertreten ist. Da die Typografie jedoch zum großen Teil durch das vorprogrammierte Schema bedingt ist, wobei der Mensch höchstens zwischen einigen, durch das System zugelassenen Formatierungsoptionen wählen kann, lässt sich dieser Aspekt nur schwer als ein Anzeichen des menschlichen Hinzutuns betrachten.

Der einzige Aspekt, wo die Rolle des Senders tatsächlich dem Menschen zuzuschreiben ist, betrifft die zweite Bedingung, die ein sprachlich kodiertes Element erfüllen muss, um das Schema zu realisieren, und zwar die sprachliche Korrektheit. Auch wenn die inhaltliche Seite der sprachlichen Einheiten, wie bereits angemerkt, keine wichtige Rolle mehr spielt, ist es nicht ohne Bedeutung, dass die darin enthaltenen Buchstaben keine zufällige Abfolge von alphabetischen Figuren sein dürfen. Der Empfänger muss beim ersten Blick imstande sein, das sprachliche Element als einen Teut einzuordnen. Eine Ausnahme wäre selbstuerständlich ein fremdsprachiges Kommunikat, bei dem der Empfänger, mangels Sprachkenntnisse, möglicherweise nicht in der Lage ist, seine sprachliche Korrektheit zu verifizieren. Auch in diesem Fall ist der Empfänger in der Regel bereit, die Einheit als einen korrekten Text zu identifizieren, indem er seine Korrektheit stillschweigend akzeptiert. 
Eine Voraussetzung ist dabei, dass die Form der Einheit in keinem Widerspruch zu dem kulturell fundierten Konzept des Tertes steht. Daraus ergibt sich, dass die sprachliche Bedeutung der Texte durch die Relation des Textseins und Tent-Nichtseins ersetzt wird. Die sprachliche Komponente des Schemas kann nämlich durch jede Einheit repräsentiert werden, die als ein Repräsentant der kulturellen Einheit „Tent“ gilt. Dies ist nicht mit dem sprachwissenschaftlichen Konzept des Textes gleichzusetzen. Während die Sprachwissenschaftler immer wieder über den linguistischen Text-Begriff diskutieren, handelt es sich dabei vielmehr um den alltäglichen Text-Begriff, der den meisten Empfängern der Massenmedien gegenwärtig ist. Wie bereits von Brinker betont wird, ist die Alltagsdefinition des Tertes ebenso nicht eindeutig:

Es lassen sich mehrere Bedeutungen des Wortes feststellen, wie "geschriebenes sprachliches Gebilde von einer gewissen Ausdehnung“, „Wortlaut“, „sprachliche Erläuterung bzw. erklärende Beschriftung“ („Unterschrift zu einer Illustration“), "Bibelstelle“, sprachlicher Teil eines musikalischen Werkes“.

BRINKER, 2010: 12

Zweifellos erfüllen die Texte in den Massenmedien relativ selten die Bedingung, die nach Klaus Brinker zu ihrer Kernbedeutung gehört, dass der Tent „in der Regel mehr als einen Satz umfasst“ (BRINKER, 2010: 12). Die wachsende Tendenz zur mosaikartigen Form bewirkt, dass die Tente überwiegend nicht länger als ein Satz oder eine satzwertige Konstruktion sind. Diese Tatsache scheint jedoch für die Empfänger aufgrund der Gewohnheit durchaus akzeptabel zu sein. Die Terte, die in den hierbei diskutierten massenmedialen Kommunikaten anwesend sind, erfüllen hingegen problemlos eine von den von Brinker angeführten alltäglichen Definitionen: „geschriebenes sprachliches Gebilde von einer gewissen Ausdehnung“ (BRINKER, 2010: 12). Demnach handelt es sich um schriftliche Einheiten, derer Ausdehnung zwar in Ausnahmefällen ein Wort, aber in der Regel ein paar Wörter umfasst. Die Bedingung, dass der Text ein sprachliches Gebilde ist, besagt, dass er explizit nach den Regeln des sprachlichen Kodes konstruiert sein muss.

Zwar erscheint diese Bedingung weiterhin als notwendig, aber es scheint auszureichen, dass ihre Realisierung nur mutmaßlich ist. Das Element „Tert“ als eine kulturelle Einheit ist unabhängig von der Sprache, in der der Tent verfasst ist, sowie von seinem sprachlich kodierten Inhalt, die bei einem fremdsprachlichen Teut für den Empfänger nicht verifizierbar sind. Die Grunduoraussetzung ist also vielmehr das Vorhandensein eines Tertes, der als solcher die Fläche füllt, die in dem Schema für einen Teut vorgesehen ist. Damit wird also einerseits die kulturelle Einheit „Tent“ signifiziert und andererseits (in globaler Hinsicht) die Vollständigkeit des Schemas mitsignifiziert, das sonst lückenhaft wäre.

\section{Schlussfolgerungen}

Die obigen Überlegungen lassen behaupten, dass der Mensch eher eine beschränkte Rolle als Produzent in den Massenmedien spielt. Seine Rolle besteht darin, in die vorher hergestellte und programmierte Maschine jeweils neue sprachlich kodierte Sequenzen von 
Buchstaben einzuführen. Auch aus dieser Perspektive unterscheidet sich die Kommunikation durch die Massenmedien deutlich von den sonstigen Formen schriftlicher Kommunikation. In den traditionelleren Formen der Massenkommunikation wie in Büchern lässt sich die Rolle des Menschen nicht unterschätzen. Dies gilt nicht nur für die Bände, die bereits vor der Erfindung Gutenbergs von einem Schreiber umgeschrieben werden mussten, sondern ebenso für die gegenwärtigen Bücher, bei denen das Geschriebene nach wie vor im Vordergrund steht. Kennzeichnend ist, dass man ausgerechnet an der gebrauchten Sprache erkennen kann, um welche Art vom Buch es sich handelt. Nach Schmitz erscheinen in jeder Kategorie von Büchern wie „Romane, Gedichtbände, Enzyklopädien“ (Schmıтz, 2004: 64) usw. „zahllose Textsorten und unterschiedliche Sprachuerwendungsweise“ (Schmıтz, 2004: 64). Obwohl sich auch Übergangsformen zwischen Büchern und Zeitschriften anzeigen lassen, beispielsweise Reiseführer oder Modejournale (ugl. Scrmıtz, 2015: 80), kann die funktionale Dominanz des Sprachlichen in den meisten Büchern als ein Argument dafür gelten, dass der Sprachstil und dadurch auch die sprachliche Komponente an sich von einer relativ großen Bedeutung sind.

In Bezug auf die Titelfrage ist festzustellen, dass sich bei der visuellen Massenkommunikation in den „neuen Medien“ nicht eindeutig ein Sender zeigen lässt. Der eine Produzent ist der Computer, der aufgrund der Vorprogrammierung die laufend eingetragenen Texte in ein fertiges Schema inskribiert. Der andere ist der Mensch, dessen Aufgabe darin besteht, die sprachlich korrekt konstruierten Texte einzutragen. Überdies wird das von dem zweiten, menschlichen Sender emittierte Kommunikat durch das „Kommunikat“ des ersten, maschinellen Senders umgeformt und teilweise reduziert. Ohne das generierte Schema hätte der sprachliche Text nämlich einen anderen Signifikationswert. Da nur einer von zwei Produzenten menschlich ist, kann man bei den besprochenen Arten der massenmedialen Kommunikation nur im beschränkten Umfang von einer Kommunikation sprechen. Ein bedeutsamer, wenn nicht der bedeutsamste Teil des Signifikationsprozesses, der das Ergebnis des gesamten Prozesses determiniert, wird auf den Computer verlagert.

\section{Literatur}

BRINKER K., 2010: Linguistische Tertanalyse. Erich Schmidt Verlag. Berlin.

Cı́szкоwsкı M., 2014: O zasadzie równoczesności $w$ multiprzekazie. W: Sawıcka G., Czechowskı W., red.: Sytuacja komunikacyjna i jej parametry (Być nadawca - Być odbiorca). Wydawnictwo Adam Marszałek. Toruń, S. 40-53.

KAPUŚCıŃSKA A., 2012: Analiza kontrastywna strategii „agenda setting” $w$ polskim dzienniku „Fakt” i niemieckim „Bild”. „Studia Linguistica“ XXXI, S. 23-36.

KAPUŚCıŃSKA A., 2016: Zum semiotischen Wert der TV-Ticker. „Zeitschrift für Literaturwissenschaft und Linguistik“ XLVI, S. 25-35.

KAPUŚCıŃSKA A., 2017: Grenzphänomene zwischen Text und Bild am Beispiel multimedialer Nachrichtensendungen. Erich Schmidt Verlag. Berlin.

KAPUŚCıŃSKA A., 2020: Technische Beschleunigung und (Des)Information. In: CiEszKowsKı M., PoCIASK J., Hrsg.: Tert- und Diskurswelten in der massenmedialen Kommunikation. Peter Lang Verlag. Berlin, S. 91-102. 
Anna Kapuścińska

McLuhan M., 1992: Die magischen Kanäle. „Understanding Media“. ECON-Verlag. Düsseldorf, Wien.

Posner, R., 1994: Terte und Kultur. In: Böhm A., Menge A., Muhr T., Hrsg.: Terte verstehen. Konzepte, Methoden, Werkzeuge (Schriften zur Informationswissenschaft). Universitätsuerlag Konstanz. Konstanz, S. 13-31.

Postman, N., 2008: Wir amüsieren uns zu Tode. Fischer Verlag. Frankfurt a. M.

Schmitz, U., 2004: Sprache in modernen Medien. Einführung in Tatsachen und Theorien, Themen und Thesen. Erich Schmidt Verlag. Berlin.

Schmitz, U., 2011: Sehflächenforschung. Eine Einführung. In: Diekmannshenke H., Klemm M., Sтӧскц H., Hrsg.: Bildlinguistik. Theorien - Methoden - Fallbeispiele. Erich Schmidt Verlag. Berlin, S. $23-42$.

Sснмітz, U., 2015: Einführung in die Medienlinguistik. Wissenschaftliche Buchgesellschaft. Darmstadt. Trojanowska, A., Francuz, P., 2007: Rozumienie przekazu audiowizualnego zawierającego, tekst taśmowy' (TV-ticker) przez osoby zależne i niezależne od pola. W: Francuz P., red.: Psychologiczne aspekty komunikacji audiowizualnej. Wydawnictwo Katolickiego Uniwersytetu Lubelskiego. Lublin, S. 67-86.

U mberto E., 1977: Zeichen. Einführung in einen Begriff und seine Geschichte. Suhrkamp Verlag. Frankfurt a. M.

U mberto E., 2002: Einführung in die Semiotik. UTB Verlag. Paderborn. 